\title{
Screening for Alcohol Misuse; Veterans Speak!
}

\author{
Jeffrey Kohlwes, MD, $\mathrm{MPH}^{1,2}$ and Patricia Cornett, $\mathrm{MD}^{1,3}$ \\ 'Internal Medicine Residency Program, University of California, San Francisco, CA, USA; '2Division of General Medicine, Veterans Affairs Medical \\ Center, San Francisco, CA, USA; ${ }^{3}$ Department of Internal Medicine, Veterans Affairs Medical Center, San Francisco, CA, USA.
}

J Gen Intern Med 30(8):1054-5

DOI: $10.1007 / \mathrm{s} 11606-015-3331-5$

(c) Society of General Internal Medicine 2015

I $\mathrm{t}$ is rare to come across a manuscript that addresses unspoken primary care physician fears and helps support what we intuitively know is the right thing to do. This month's JGIM features such an article. In their manuscript, Simonetti and colleagues ${ }^{1}$ demonstrate a positive correlation between patient satisfaction and the receipt of a brief alcohol screening at a primary care clinic visit. They performed a cross-sectional, secondary data analysis using the Veterans Affairs Survey of Healthcare Experiences of Patients (SHEP) data to examine if, in veterans with unhealthy alcohol use, outpatient alcohol misuse screening changed patient reported quality of care. To screen for alcohol misuse, the authors used the well-validated Alcohol Use Disorder Identification Test Consumption Questionnaire (AUDIT-C). Nearly $50 \%$ of the veterans were screened, and reassuringly, a higher proportion of veterans who reported receipt of the brief screening intervention rated their provider more favorably than those who were not screened.

While our fiduciary responsibility to our patients is to serve as a trusted professional, most of us also want our patients to like us, and we worry that if we ask about "blank" (fill in: substance abuse, alcohol misuse, sexually transmitted disease screening, seat belt use, immunizations, etc.) they may be embarrassed and not want to return. Now, with patient satisfaction surveys and 360-degree evaluations becoming more prominent feedback tools and increasingly being tied to reimbursement, these concerns with patient satisfaction are even more relevant for trainees and practicing physicians alike. However, the findings of Simonetti and colleagues seems a clear indication that our patients don't simply want to like us, but expect us to do our job even if it means addressing difficult-to-talk-about topics such as alcohol misuse. The authors appropriately conclude that screening for alcohol misuse does not adversely affect patients' perception of their quality of care and, in fact, increases it.

This study does not address another question commonly asked in the primary care setting: What do I do

Published online April 22, 2015 now that my patient has screened positive for alcohol misuse? Although patient satisfaction increases with screening, primary care provider satisfaction likely decreases when receiving a positive screen. ${ }^{2}$ Many may feel like they have poked a skunk and don't like the smell. Therefore, it is important to not just screen for alcohol use disorder, but to have a clear plan of action for a positive screen. Data supports that just bringing up alcohol cessation in primary care promotes abstinence from problem drinking, which is improved further with a brief intervention after a positive screen. ${ }^{3}$ But most providers remain uneasy with addressing maladaptive behaviors with a brief intervention if they don't possess the skills to intervene. Current leaders in primary care who are involved in such efforts as the VA Centers of Excellence or Patient Centered Medical Homes emphasize a multi-provider approach to patient care where warm handoffs to behavioral health specialists can facilitate addressing substance abuse issues for willing patients. ${ }^{4}$ These excellent models have not been sufficiently adopted by most primary care systems to replace the need for front-line practioners being able to address positive screens on their own.

Several models exist, however, to empower primary care practioners to help their patients change their behavior. One tool that is important for clinicians to have in their armamentarium is motivational interviewing (MI). MI utilizes a collaborative conversation style for strengthening a person's own motivation and commitment to change. Using the OARS approach with Openended questions, Affirmations, Reflections and Summary statements, primary care providers can engage their patients in an open and supportive way to help move them through the stages of change. ${ }^{5}$ Simultaneously, MI can help physicians feel like they have the tools not only to screen, but to intervene. MI is one of the skills used in another very high quality resource: the Substance Use in Adults and Adolescents: Screening, Brief Intervention and Referral to Treatment (SBIRT) program. This indispensable training program is offered through the Substance Abuse and Mental Health Services Administration (SAMHSA). This free online course offers skills for primary care practioners to recognize and address substance abuse in their offices. There are also more advanced courses for practitioners who devote a significant amount of time to substance abuse screening and treatment. ${ }^{6}$ 
Finally, another important outcome of this study was the measurement of the overall satisfaction numbers shown by the VA study patients who had screened positive for alcohol misuse. Recently, there have been several well-publicized scandals involving the VA that have led to a dip in morale as faculty and staff feel let down by a small number of poorly performing employees. The perception portrayed in the media is that care at the VA is substandard. ${ }^{7}$ This perception is strengthened further by legislation encouraging veterans to seek care outside the VA rather than supporting our existing programs. The Simonetti study demonstrates impressive satisfaction numbers among the VA patients enrolled in the study. Amongst the patients that were screened for alcohol misuse, nearly $83 \%$ of veterans rated their VA care as high quality. Patients screening positive for substance abuse may be more disenfranchised and more prone to lower healthcare satisfaction, making this figure all the more impressive. Similarly, the reported $87 \%$ satisfaction score that Veterans reported with their providers is in the 75 th percentile of satisfaction on the AHRQ Clinician \& Group Survey (CG-CAHPS) website. ${ }^{8}$ As devoted VA clinicians, we are not surprised by these findings, although it is a welcome validation of the hard work and dedication shown by our colleagues.
Corresponding Author: Jeffrey Kohlwes, MD, MPH; Internal Medicine Residency Program, University of California, San Francisco, CA 94121, USA (e-mail: Jeff.Kohlwes@ucsf.edu).

\section{REFERENCES}

1. Simonetti JA, Lapham GT, Williams EC. Association between receipt of brief alcohol intervention and quality of care among Veteran outpatients with unhealthy alcohol use. J Gen Intern Med. 2015

2. Park ER, Wolfe TJ, Gokhale M, Winickoff JP, Rigotti NA. Perceived preparedness to provide preventive counseling: reports of graduating primary care residents at academic health centers. J Gen Intern Med. 2005;20(5):386-391.

3. U.S. Preventive Services Task Force. Screening and behavioral counseling interventions in primary care to reduce alcohol misuse: recommendation statement. Ann Intern Med. 2013;159(3):210-218.

4. Gilman SC, Chokshi DA, Bowen JL, Rugen KW, Cox M. Connecting the dots: interprofessional health education and delivery system redesign at the Veterans Health Administration. Acad Med. 2014;89(8):1113-1116.

5. Söderlund LL, Madson MB, Rubak S, Nilsen P. A systematic review of motivational interviewing training for general health care practitioners. Patient Educ Couns. 2011;84(1):16-26.

6. http://www.integration.samhsa.gov/clinical-practice/sbirt/training-otherresources. Accessed March 6th, 2015

7. http://www.cbsnews.com/feature/va-hospitals-scandal/. Accessed March 6th, 2015

8. https://cahpsdatabase.ahrq.gov/CAHPSIDB/Public/CG/CG_Topscores. aspx. Accessed March 6th, 2015 